\title{
Review
}

\section{Stem cells and small molecule screening: haploid embryonic stem cells as a new tool}

\author{
Bi WU ${ }^{1,2}$, Wei $\mathrm{LI}^{2, *}$, Liu WANG ${ }^{2}$, Zhong-hua LIU ${ }^{1}$, Xiao-yang ZHAO ${ }^{2, *}$ \\ ${ }^{1}$ College of Life Science, Northeast Agricultural University of China, Harbin 150030, China; ${ }^{2}$ State Key Laboratory of Reproductive \\ Biology, Institute of Zoology, Chinese Academy of Sciences, Beijing 100101, China
}

\begin{abstract}
Stem cells can both self-renew and differentiate into various cell types under certain conditions, which makes them a good model for development and disease studies. Recently, chemical approaches have been widely applied in stem cell biology by promoting stem cell self-renewal, proliferation, differentiation and somatic cell reprogramming using specific small molecules. Conversely, stem cells and their derivatives also provide an efficient and robust platform for small molecule and drug screening. Here, we review the current research and applications of small molecules that modulate stem cell self-renewal and differentiation and improve reprogramming, as well as the applications that use stem cells as a tool for small molecule screening. Moreover, we introduce the recent advance in haploid embryonic stem cells research. Haploid embryonic stem cells maintain haploidy and stable growth over extensive passages, possess the ability to differentiate into all three germ layers in vitro and in vivo, and contribute to the germlines of chimeras when injected into blastocysts. Androgenetic haploid stem cells can also be used in place of sperm to produce fertile progeny after intracytoplasmic injection into mature oocytes. Such characteristics demonstrate that haploid stem cells are a new approach for genetic studies at both the cellular and animal levels and that they are a valuable platform for future small molecule screening.
\end{abstract}

Keywords: small molecules; stem cells; haploid embryonic stem cells; induced pluripotent stem cell (iPS); drug screening

Acta Pharmacologica Sinica (2013) 34: 725-731; doi: 10.1038/aps.2013.26; published online 6 May 2013

\section{Introduction}

Stem cells are found in all multi-cellular organisms and can both self-renew and differentiate into diverse specialized cell types. In mammals, in vitro cultured stem cells can be classified into two types according to their differentiation ability; these types are pluripotent and multipotent stem cells. Pluripotent stem cells mainly include embryonic stem (ES) cells and induced pluripotent stem (iPS) cells. ES cells are derived from the inner cell mass of blastocysts. iPS cells are directly reprogrammed from somatic cells by the ectopic expression of specific factors. The cells can both self-renew indefinitely in vitro and differentiate into any cell type that is representative of all three germ layers under the appropriate conditions both in vivo and in vitro ${ }^{[1,2]}$. Multipotent stem cells mainly include various types of adult stem cells. Adult stem cells act as a repair system that the body can use to replenish old or bad tissues in adult organisms. These characteristics indicate that stem cells are a good model to use to study the process

\footnotetext{
* To whom correspondence should be addressed.

E-mail xyzhao@ioz.ac.cn (Xiao-yang ZHAO); liwei@ioz.ac.cn (Wei LI)

Received 2013-01-24 Accepted 2013-03-05
}

of organismal development and disease and that they are an important tool ${ }^{[3]}$ in tissue engineering, regenerative medicine, and drug screening ${ }^{[4]}$.

Small molecules are emerging as useful chemical tools in biomedical research and applications. Through modulating specific targets in biological signaling pathways or exerting epigenetic modifications on the genome, small molecules can modify or even change cell fate ${ }^{[5,6]}$. For example, small molecules can maintain ES cells in the pluripotent state by inhibiting the differentiation pathway. Conversely, stem cells have been widely utilized in small molecule screening.

Here, we review the research and application of small molecules in stem cell biology, including reprogramming, stem cell self-renewal and differentiation. We also introduce the recent advances in the use of haploid embryonic stem cells, which is a powerful tool for functional genomics studies and small molecule screening, and discuss the future application of haploid ES cells in small molecule and drug screening.

\section{The small molecules that modulate stem cells Stem cell self-renewal}

In conventional culture systems, ES cells in the undifferentiated state are maintained on feeder cells in medium that con- 
tains components such as animal serum or serum replacement with cytokines (Table 1). These media can keep ES cells in an undifferentiated state for many passages, however, it also increases the complexity of determining the molecular processes that regulate ES cell self-renewal. Currently, small molecules can be synthesized that enable the formulation of nonanimal-derived chemically defined culture systems, which, in turn, enable the growth of ES cells in xeno-free conditions for therapeutic applications.

Table 1. Factors used for maintaining ES cell self-renewal.

\begin{tabular}{llll}
\hline Factor & Cell type & $\begin{array}{c}\text { Target signal } \\
\text { pathway }\end{array}$ & \multicolumn{1}{c}{ Function } \\
\hline LIF & mES & STAT3 & $\begin{array}{l}\text { Active STAT3, self-renew } \\
\text { Inhibit ERK, self-renew }\end{array}$ \\
BMP4 & mES & ERK & $\begin{array}{l}\text { Differentiate } \\
\text { hES }\end{array}$ \\
bFGF & hES & ERK & $\begin{array}{l}\text { Self-renew } \\
\text { Self-renew }\end{array}$ \\
Activin A & hES & SMAD & S \\
\hline
\end{tabular}

mES, mouse embryonic stem; hES, human embryonic stem.

Small molecules can simply inhibit differentiation to maintain ES cell self-renewal. This view is supported by a recent study showing that CHIR99021 (Table 2) acts via the inhibition of glycogen synthase kinase 3 (GSK3) to enhance ES-cell growth capacity and viability ${ }^{[7]}$. GSK3 has been implicated in regulating both self-renewal and differentiation ${ }^{[8]}$. Wnt signaling inhibits GSK3 and stabilizes cytoplasmic $\beta$-catenin ( $\beta$-Ctnn). CHIR99021 prevents the phosphorylation of $\beta$-Ctnn by GSK3 $\beta$ and activates Wnt signaling. The mitogen-activated protein kinase (MEK/EKR) pathway induces mouse embryonic stem cell (mES cells) differentiation. MEK inhibitor PD0325901 (Table 2) can inhibit the MEK signaling pathway to promote mouse embryonic stem (mES) cell self-renewal ${ }^{[7]}$. Chen et al reported that retinol (vitamin A) (Table 2) can support the feeder-independent self-renewal of $\mathrm{mES}$ cells in longterm culture by modulating the expression of $\operatorname{Nanog}^{[9]}$.

Small molecules also provide a new way for generating pluripotent cell lines from other species. For example, CHIR99021 and PD0325901 cause rat embryonic stem (rES) cells to self-renew and maintain their pluripotent states ${ }^{[10,11]}$. As we known, human embryonic stem (hES) cells are different from mES cells in gene expression and self-renewal signaling pathways. mES cells can be maintained by leukemia inhibitory factor (LIF) ${ }^{[12]}$ and bone morphogenetic protein (BMP), while hES cells self-renew under basic fibroblast growth factor $(\mathrm{bFGF})^{[2]}$ and Activin A (Table 1). Recently, a study reported that hES cells could be stably maintained under a combination of bFGF, CHIR99021 and PD0325901 (Table 1) ${ }^{[13]}$. Another human pluripotent cell type, mESC-like human iPS cells (m-hiPSs), can stably self-renew when the culture medium contains CHIR99021, PD0325901 and A8301 (a small molecule inhibitor of the transforming growth factor $\beta$ (TGF $\beta$ )/Activin receptors) (Table 2).

\section{Stem cell differentiation}

Differentiation is the process during which unspecialized cells become specialized and functional cells with restricted developmental potential. Traditionally, embryoid bodies (EBs) were formed first, and the desired cells were then isolated from the culture. This method is ineffective and time-consuming. Currently, chemically defined medium containing small molecules can be used directly to complete the differentiation process. We focus here on some strategies for using small molecules to differentiate ES cells into specific lineages.

In a recent study, a number of small molecules have been identified that can regulate the tissue-specific differentiation of ES cells. For example, the molecules IDE1 and IDE2 (Table 2) have been identified as effective inducers of definitive endoderm ${ }^{[14]}$. Similar to Activin A and Nodal (a natural inducer of definitive endoderm), both IDE1 and IDE2 induce Smad2 phosphorylation in mES cells through unknown targets. Retinoic acid (RA) followed by Sonic hedgehog (Shh) or the Shh agonist Hh-Ag1.3 induced mES and hES cells to differentiate into motor neurons (Table 2). Moreover, Li et al found that hES cells can be differentiated into motor neurons efficiently by the simple sequential application of RA and $S_{h} h^{[15]}$. The authors also discovered that the small molecule purmorphamine, a purine derivative that activates the Shh pathway, could replace Shh to generate motor neurons in a similar way. Sulfonyl-hydrazones (Shz) (Table 2) potently induce Nkx2.5 and a subset of other cardiac markers in a variety of stem/progenitor cells ${ }^{[16]}$, but their efficiency is quite low and the process is not completely understood.

\section{Reprogramming}

Induced pluripotent stem (iPS) cells generated from mouse somatic cells by the ectopic expression of four defined genetic factors provide a new approach for epigenetic reprogramming ${ }^{[17]}$. It has been reported that rhesus monkey ${ }^{[18]}$ and human ${ }^{[19]}$ somatic cells can also be reprogrammed to iPS cells through the viral introduction of four transcription factors: Oct4, Sox2, Klf4, and c-Myc or Oct4, Sox2, Lin28, and Nanog. These iPS cells not only possess morphological and molecular similarities to ES cells, but they also hold the same developmental potentials as ES cells. This method gives us an opportunity to generate patient-specific cells for therapeutic application. However, the method raises another question about whether it is safe to use retrovirus-mediated iPS cells, the use of which comes with the risk of causing tumors.

Although iPS cells have been generated through viral and non-viral methods, the reprogramming is still a slow and inefficient process. Some studies have found that small molecules can facilitate reprogramming and improve reprogramming efficiency. For instance, Huangfu et al discovered that the reprogramming of mouse embryonic fibroblasts (MEFs) to iPS cells could be promoted by the DNA demethylating agent 5-azacytidine and the histone deacetylase inhibitors suberoylanilide hydroxamic acid, trichostatin A and VPA (Table 2) ${ }^{[20]}$. 
A small-molecule inhibitor of the histone methyltransferase G9a, BIX-01294, also enabled the reprogramming of neural progenitor cells (NPCs) transduced with Sox2/Klf4/c-Myc without Oct4. Because Oct4 is repressed by G9a, the repression is alleviated via G9a repression by BIX-01294, which results in Oct4-independent reprogramming ${ }^{[21]}$. In addition to these direct epigenetic modulators, the dual inhibition of MEK and TGF $\beta$ by PD0325901 and SB431542 (Table 2) can also dramatically improve (>100-fold) the generation of iPS cells from human fibroblasts with an efficiency of $>1 \%$ by enhancing the mesenchymal-epithelial transition ${ }^{[22]}$.

\section{Stem cells as a tool for small molecule screening}

One of the most valuable functions of stem cells is modeling human diseases. Stem cell-derived cell types can be used to establish cell-based in vitro test systems for drug screening, toxicity testing, and functional analysis. These characteristics make stem cells a powerful tool for drug screening.

Screening small molecule drugs has been utilized in three types of cells: embryonic stem cells, cancer stem cells, and patient-specific induced pluripotent stem cells. Highthroughput screening (HTS) for the development of new drugs with such stem cells has been widely adopted to more effectively screen for drugs for diseases such as Parkinson's disease or cancers. For example, Takahashi et al used ES cells that were stably transfected with cardiac-specific a-cardiac myosin heavy chain (MHC) promoter-driven enhanced green fluorescent protein (EGFP) as a tool for drug screening. Eight hundred eighty compounds approved for human use were screened for their ability to induce cardiac differentiation. Fluorescence microscopic analysis showed that vitamin C (Table 2) can significantly induce ES cells to undergo myocardial cell differentiation ${ }^{[23]}$. Visnyei et al described a high-throughput small molecule screening approach that can identify and characterize chemical compounds that are effective against glioblastoma (GBM) stem cells ${ }^{[24]}$. The authors found that compounds \#5560509 (Table 2) and \#5256360 (Table 2) inhibited the expression of the mitotic module genes. Lumelsky et al found that nicotinamide can induce mouse ES cells into insulin-secreting cells with an insulin secretion adjustment mechanism similar to that of normal beta cells ${ }^{[25]}$. Nichols et al found that the small molecules Y-27632 and H-1152 can inhibit LRRK2 because they inhibit the Rho kinase (ROCK) ${ }^{[26]}$. In contrast to this finding, it was reported that GSK429286A inhibits ROCK but does not inhibit LRRK2 $2^{[27]}$.

These studies may help in understanding the pathogenesis of Parkinson's disease and the development of new treatments ${ }^{[26]}$. The authors of a recent study also showed that they, with the NIH Molecular Libraries Program, optimized a compound as a probe (ML239) that displayed greater than 20-fold selective inhibition of the breast cancer stem cell-like cell line HMLE_sh_Ecad over the isogonic control line HMLE_ sh_GFP ${ }^{[28]}$. Additionally, SKF-86466 was found to induce IKBKAP (the gene responsible for familial dysautonomia) transcription through the modulation of intracellular cAMP levels and PKA-dependent CREB phosphorylation ${ }^{[29]}$. These studies all suggest that stem cells can be an important tool for new small molecule/drug screening.

\section{Mammalian haploid cells}

Most organisms have diploid chromosomes; only rare species, such as yeast and bees, have haploid chromosomes. With the aim to determine all gene functions, haploid cells have obvious advantages because they carry only a single set of chromosomes, thus bypassing allelic affections. However, in mammals, haploid cells only exist in germ cells and are occasionally found in tumors. Previous reports showed that haploid cells can be derived in fish ${ }^{[30]}$ and human KBM-7 leukemia cells $^{[31,32]}$. Recently, mouse haploid embryonic stem (hES) cells have been successfully isolated from both parthenogenetic ${ }^{[33]}$ and androgenetic embryos ${ }^{[34,35]}$, which are all cultured in $2 \mathrm{i}$ medium (Figure 1) ${ }^{[7]}$. These hES cells have stem cell morphology, maintain a haploid karyotype, express all of the stem cell marker genes (eg, Oct4, Nanog, Sox2, and SSEA-1), and have the ability to differentiate into the cell types of all three germ layers, including a contribution to chimeric mice and the germline. Furthermore, as haploid stem cells have a haploid karyotype, they can take the place of sperm to "fertilize" eggs by intracytoplasmic injection into oocytes and produce fertile offspring ${ }^{[34,35]}$.

Haploid cells combine haploidy and pluripotency well and have broad applications in functional genomics studies, as well as in both genetic and small molecule screening. Due to the absence of a second gene copy in haploid stem cells, homozygous mutations can be easily achieved. Because haploid ES cells can be easily propagated, genome-wide mutation libraries can be established by gene trapping. This development provides a fast and simple approach for genetic studies ${ }^{[36]}$. Moreover, the genetic studies performed in cells can be easily transferred to animals by intracytoplasmic androgenetic haploid ES cell injection (ICAI) into oocytes.

In the normal process of fertilization, both paternal and maternal imprints are needed to form a normal organism. A lack of any parental imprints will compromise embryonic development ${ }^{[37,38]}$. Haploid stem cells from parthenogenetic blastocysts may not support the full-term development of embryos after injection into MII oocytes due to the parthenogenetic nature of the reconstructed embryos. Conversely, androgenetic haploid stem cells may support the full-term development of embryos after injection into MII oocytes if the haploid stem cells maintain the paternal imprints ${ }^{[34]}$. Indeed, the DMRs of two maternally imprinted genes, Snrpn and Airn, kept their unmethylated status at both early and late cell passages, as in sperm; whereas paternally imprinted genes (H19 and Gtl2) showed inconsistent methylation statuses, with the tendency to keep a sperm-like methylation status in early cell passages and to partially lose methylation during passaging ${ }^{[34]}$.

We chiefly introduce the derivation of mouse haploid stem cells here. For parthenogenetic haploid ES cell derivation, the mouse parthenogenetic blastocysts are chosen and placed onto feeders in chemically defined $2 \mathrm{i}$ medium plus LIF as described $^{[7]}$. For androgenetic haploid ES cell derivation, a 
Table 2. Small molecules that modulate ES cell self-renewal, differentiation and somatic cell reprogramming.

\begin{tabular}{|c|c|c|c|}
\hline Molecule & Name & Targets & Effect \\
\hline & CHIR99021 & GSK3 & ES self-renew \\
\hline & PD0325901 & MEK & Prevent differentiation \\
\hline & IDE1 & Unknown & Induces endoderm differentiation \\
\hline $\mathrm{HO}^{-}$ & IDE2 & Unknown & Induces endoderm differentiation \\
\hline & BIX-01294 & G9a-HMTASE & Reprogramming of NPCs and MEFs \\
\hline & VPA & Histone deacetylase & Promotes reprogramming \\
\hline & SB431542 & ALK5, ALK4 and ALK7 & $\begin{array}{l}\text { Induces neural differentiation of } \\
\text { WithNoggin }\end{array}$ \\
\hline & A8301 & ALK5, ALK4 and ALK7 & $\begin{array}{l}\text { Supports riPS cell self-renewal with } \\
\text { CHIR99021 and PD0325901 }\end{array}$ \\
\hline & 5-azacytidine & DNA methylationinhibition & $\begin{array}{l}\text { Human ESC differentiation, } \\
\text { reprogramming }\end{array}$ \\
\hline & Kenpaullone & CDKs and GSK3 & Induce MEF reprogramming \\
\hline & \#5560509 & MELKASPMTOP2AFOXM1b & Inhibitmitotic module genes \\
\hline & \#5256360 & & \\
\hline
\end{tabular}




Effect
ES cell neuronal differentiation

A

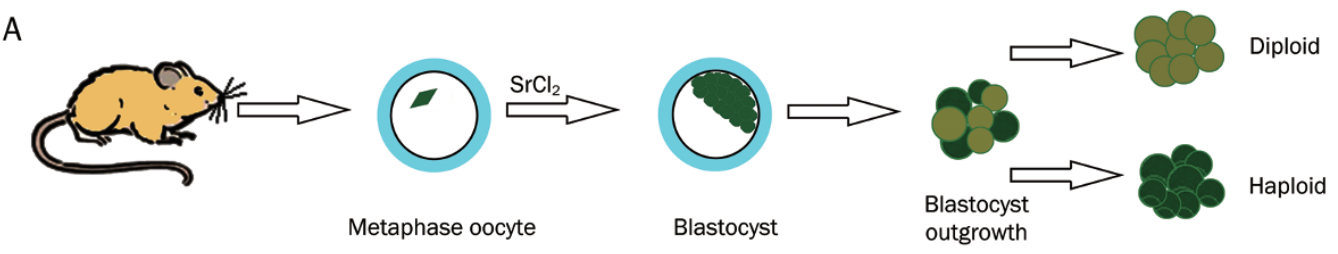

B

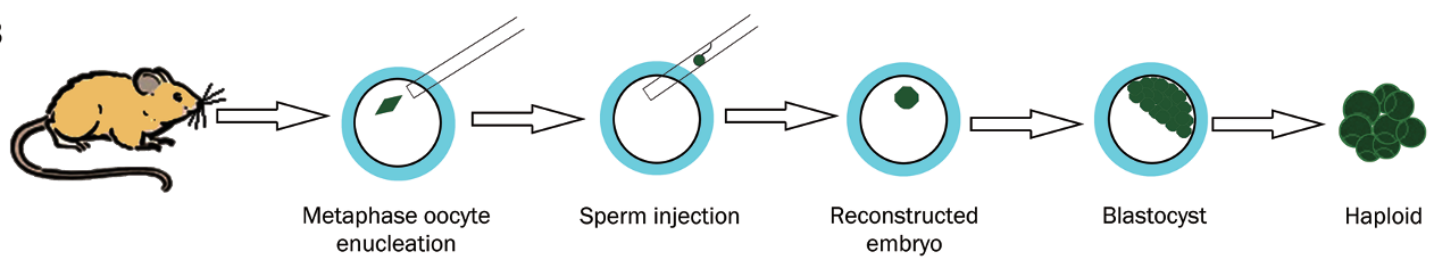

C

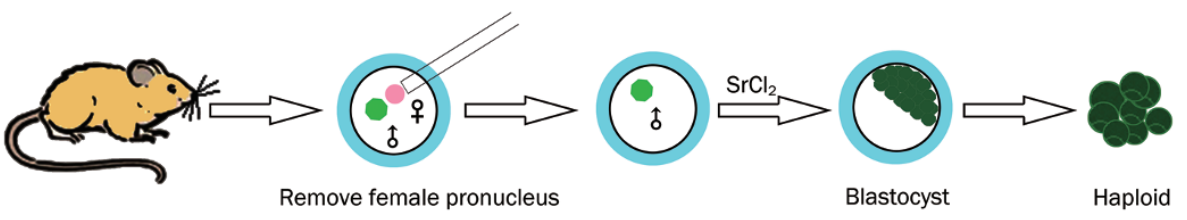

Figure 1. Generation of haploid embryonic stem cells. (A) Diagram for the generation of parthenogenetic haploid embryonic stem cells. (B) Diagram for the generation of ahES cells from androgenetic blastocysts. (C) Diagram for the generation of ahESC cells by remove female pronucle from zygote.

haploid sperm head is directly injected into an enucleated oocyte, or the female pronucleus is removed from fertilized oocytes to construct the haploid embryos. The recovered haploid morulas/blastocysts are cultured in $2 \mathrm{i}$ medium for cell line derivation. The cell line derivation process is similar to diploid ES cell line derivation. However, FACS-mediated haploid cell purification and propagation are needed to overcome the dynamic diploidization of haploid ES cells. Purification by FACS sorting every four to five passages can maintain a high proportion of haploid ES cells in the cultured cell lines.

Two different approaches can be used to produce offspring from haploid ES cells. One approach is to use haploid $G_{0} / G_{1}$ phase androgenetic haploid ES cells for oocyte injection, a procedure similar to intracytoplasmic sperm injection (ICSI), or round sperm injection (ROSI). The other approach is to use metaphase androgenetic haploid ES cells for oocyte injection, a procedure similar to the 'semi-cloning' approach.

Taken together, these results demonstrate that haploid embryonic stem cells can combine haploidy and pluripotency and become powerful tools for use in functional genomics studies. Moreover, androgenetic haploid embryonic stem cells could take the place of sperm to fuse with oocytes and produce fertile offspring, thus providing a new approach for transgenic mice production ${ }^{[39]}$. 


\section{Small molecule screening with haploid stem cells}

Previous studies show that it is possible to generate genomewide mutagenesis libraries with haploid embryonic stem cells because they lack one set of chromosomes. This finding suggests that haploid stem cells could be a convenient tool for genetic studies and drug screening. By generating cell libraries with genome-wide genetic mutations, combined with highthroughput screening technology, robust and rapid screening for small molecules or drugs that specifically target genes and pathways can be efficiently performed. This process has already been tested on parthenogenetic embryonic stem cells by Elling et al through retrovirus-based gene trapping: they generated haploid ES cell libraries containing millions of different insertional mutations. Then, they performed a forward genetic screen for ricin toxicity, a dangerous poison that may be used as a biological weapon ${ }^{[36]}$. The screen revealed that the G Protein-Coupled Receptor (GPCR) Gpr107 is an essential molecule required for ricin-induced killing because Gpr107 mutation in haploid ES cells conferred resistance to ricin toxicity. This study suggests that future screening for antitoxins to ricin may be focused on molecules that inhibit Gpr107.

It is also possible to screen small molecules that modulate genome imprinting because the gamete-derived haploid stem cells maintain partial genomic imprinting. Imprinting plays an important role in mammalian embryonic development. The abnormal regulation of imprinted genes can cause many human diseases ${ }^{[40,41]}$, such as Beckwith-Wiedemann syndrome (BWS), Russell-Silver syndrome (RSS), and Angelman syndrome (AS). Small molecules that regulate the imprinting status in haploid cells may be able to regulate the developmental processes and cure the diseases associated with imprinted genes.

Here, we propose a general procedure for using haploid ES cells to screen for small molecules that may regulate development or cure diseases. First, generate a haploid ES cell line that carries selection markers suitable for high-throughput screening, and then use this cell line to generate ES cell libraries containing genome-wide homozygous genetic mutations. For example, use the retrovirus- or piggyBac transposonbased gene trap system to create large-scale insertional mutagenesis. Second, choose a chemical library that is relevant to the question being addressed for screening. There are many established chemical libraries, and a specific chemical library such as kinase inhibitors can be used based on the topic under investigation. Third, identify the small molecules that hit the target according to the readout. Finally, confirm the functions of the small molecules with more cellular or animal testing.

\section{Summary}

In this review, we briefly introduce interdisciplinary studies on small molecules and stem cell biology. These studies have clearly demonstrated that small molecules are important tools in modulating stem cell fate, while stem cells are valuable platforms for small molecule screening. In addition to the established approaches, new tools such as haploid stem cells are emerging for future research. Haploid stem cells can pro- duce genome-wide homozygous mutation in an efficient and robust way and hold the potential to transmit these mutations into animals. Such characteristics make them a convenient approach for genetic screening, which will also provide molecular targets for small molecule screenings. We anticipate that in the future, stem cells, especially haploid embryonic stem cells, will constitute basic platforms for efficiently screening specific small molecules, which can subsequently efficiently modulate cell fate or cure diseases.

\section{Acknowledgements}

This work was supported by grants 2012CB966500 (to Xiaoyang ZHAO) and 2011CB965301 (to Liu WANG) from the China National Basic Research Program.

\section{References}

1 Martin GR. Isolation of a pluripotent cell line from early mouse embryos cultured in medium conditioned by teratocarcinoma stem cells. Proc Natl Acad Sci U S A 1981; 78: 7634-8.

2 Thomson JA, Itskovitz-Eldor J, Shapiro SS, Waknitz MA, Swiergiel JJ, Marshall VS, et al. Embryonic stem cell lines derived from human blastocysts. Science 1998; 282: 1145-7.

3 Capecchi MR. Altering the genome by homologous recombination. Science 1989; 244: 1288-92.

4 Sartipy P, Bjorquist P, Strehl R, Hyllner J. The application of human embryonic stem cell technologies to drug discovery. Drug Discov Today 2007; 12: 688-99.

5 Schugar RC, Robbins PD, Deasy BM. Small molecules in stem cell self-renewal and differentiation. Gene Ther 2008; 15: 126-35.

$6 \mathrm{Xu}$ Y, Shi Y, Ding S. A chemical approach to stem-cell biology and regenerative medicine. Nature 2008; 453: 338-44.

7 Ying QL, Wray J, Nichols J, Batlle-Morera L, Doble B, Woodgett J, et al. The ground state of embryonic stem cell self-renewal. Nature 2008; 453: 519-23.

8 Bone HK, Damiano T, Bartlett S, Perry A, Letchford J, Ripoll YS, et al. Involvement of GSK-3 in regulation of murine embryonic stem cell self-renewal revealed by a series of bisindolylmaleimides. Chem Biol 2009; 16: 15-27.

9 Chen L, Khillan JS. Promotion of feeder-independent self-renewal of embryonic stem cells by retinol (vitamin A). Stem Cells 2008; 26 : 1858-64.

10 Buehr M, Meek S, Blair K, Yang J, Ure J, Silva J, et al. Capture of authentic embryonic stem cells from rat blastocysts. Cell 2008; 135 : 1287-98.

11 Li P, Tong C, Mehrian-Shai R, Jia L, Wu N, Yan Y, et al. Germline competent embryonic stem cells derived from rat blastocysts. Cell 2008; 135: 1299-310.

12 Niwa H, Burdon T, Chambers I, Smith A. Self-renewal of pluripotent embryonic stem cells is mediated via activation of STAT3. Genes Dev 1998; 12: 2048-60.

13 Tsutsui H, Valamehr B, Hindoyan A, Qiao R, Ding X, Guo S, et al. An optimized small molecule inhibitor cocktail supports long-term maintenance of human embryonic stem cells. Nat Commun 2011; 2 : 167.

14 Borowiak M, Maehr R, Chen S, Chen AE, Tang W, Fox JL, et al. Small molecules efficiently direct endodermal differentiation of mouse and human embryonic stem cells. Cell Stem Cell 2009; 4: 348-58.

15 Li XJ, Hu BY, Jones SA, Zhang YS, Lavaute T, Du ZW, et al. Directed differentiation of ventral spinal progenitors and motor neurons from 
human embryonic stem cells by small molecules. Stem Cells 2008; 26: 886-93.

16 Sadek H, Hannack B, Choe E, Wang J, Latif S, Garry MG, et al. Cardiogenic small molecules that enhance myocardial repair by stem cells. Proc Natl Acad Sci U S A 2008; 105: 6063-8.

17 Takahashi K, Yamanaka S. Induction of pluripotent stem cells from mouse embryonic and adult fibroblast cultures by defined factors. Cell 2006; 126: 663-76.

18 Liu H, Zhu F, Yong J, Zhang P, Hou P, Li H, et al. Generation of induced pluripotent stem cells from adult rhesus monkey fibroblasts. Cell Stem Cell 2008; 3: 587-90.

19 Takahashi K, Tanabe K, Ohnuki M, Narita M, Ichisaka T, Tomoda K, et al. Induction of pluripotent stem cells from adult human fibroblasts by defined factors. Cell 2007; 131: 861-72.

20 Huangfu D, Maehr R, Guo W, Eijkelenboom A, Snitow M, Chen AE, et al. Induction of pluripotent stem cells by defined factors is greatly improved by small-molecule compounds. Nat Biotechnol 2008; 26: 795-7.

21 Feldman N, Gerson A, Fang J, Li E, Zhang Y, Shinkai Y, et al. G9amediated irreversible epigenetic inactivation of Oct-3/4 during early embryogenesis. Nat Cell Biol 2006; 8: 188-94.

22 Lin T, Ambasudhan R, Yuan X, Li W, Hilcove S, Abujarour R, et al. A chemical platform for improved induction of human iPSCs. Nat Methods 2009; 6: 805-8.

23 Takahashi T, Lord B, Schulze PC, Fryer RM, Sarang SS, Gullans SR, et al. Ascorbic acid enhances differentiation of embryonic stem cells into cardiac myocytes. Circulation 2003; 107: 1912-6.

24 Visnyei K, Onodera H, Damoiseaux R, Saigusa K, Petrosyan S, De Vries $\mathrm{D}$, et al. A molecular screening approach to identify and characterize inhibitors of glioblastoma stem cells. Mol Cancer Ther 2011; 10 : 1818-28.

25 Lumelsky N, Blondel O, Laeng P, Velasco I, Ravin R, McKay R. Differentiation of embryonic stem cells to insulin-secreting structures similar to pancreatic islets. Science 2001; 292: 1389-94.

26 Nichols RJ, Dzamko N, Hutti JE, Cantley LC, Deak M, Moran J, et al. Substrate specificity and inhibitors of LRRK2, a protein kinase mutated in Parkinson's disease. Biochem J 2009; 424: 47-60.

27 Goodman KB, Cui H, Dowdell SE, Gaitanopoulos DE, Ivy RL, Sehon CA, et al. Development of dihydropyridone indazole amides as selective Rho-kinase inhibitors. J Med Chem 2007; 50: 6-9.
28 Germain AR, Carmody LC, Morgan B, Fernandez C, Forbeck E, Lewis TA, et al. Identification of a selective small molecule inhibitor of breast cancer stem cells. Bioorg Med Chem Lett 2012; 22: 3571-4.

29 Lee G, Ramirez CN, Kim H, Zeltner N, Liu B, Radu C, et al. Large-scale screening using familial dysautonomia induced pluripotent stem cells identifies compounds that rescue IKBKAP expression. Nat Biotechnol 2012; 30: 1244-8.

30 Yi M, Hong N, Hong Y. Generation of medaka fish haploid embryonic stem cells. Science 2009; 326: 430-3.

31 Carette JE, Guimaraes CP, Varadarajan M, Park AS, Wuethrich I, Godarova A, et al. Haploid genetic screens in human cells identify host factors used by pathogens. Science 2009; 326: 1231-5.

32 Carette JE, Raaben M, Wong AC, Herbert AS, Obernosterer G, Mulherkar $\mathrm{N}$, et al. Ebola virus entry requires the cholesterol transporter Niemann-Pick C1. Nature 2011; 477: 340-3.

33 Leeb M, Wutz A. Derivation of haploid embryonic stem cells from mouse embryos. Nature 2011; 479: 131-4.

34 Li W, Shuai L, Wan H, Dong M, Wang M, Sang L, et al. Androgenetic haploid embryonic stem cells produce live transgenic mice. Nature 2012; 490: 407-11.

35 Yang H, Shi L, Wang BA, Liang D, Zhong C, Liu W, et al. Generation of genetically modified mice by oocyte injection of androgenetic haploid embryonic stem cells. Cell 2012; 149: 605-17.

36 Elling U, Taubenschmid J, Wirnsberger G, O'Malley R, Demers SP, Vanhaelen $\mathrm{Q}$, et al. Forward and reverse genetics through derivation of haploid mouse embryonic stem cells. Cell Stem Cell 2011; 9: 563-74.

37 Surani MA, Barton SC. Development of gynogenetic eggs in the mouse: implications for parthenogenetic embryos. Science 1983; 222: $1034-6$.

38 Surani MA, Barton SC, Norris ML. Development of reconstituted mouse eggs suggests imprinting of the genome during gametogenesis. Nature 1984; 308: 548-50.

39 Leeb M, Walker R, Mansfield B, Nichols J, Smith A, Wutz A. Germline potential of parthenogenetic haploid mouse embryonic stem cells. Development 2012; 139: 3301-5.

40 Weksberg R. Imprinted genes and human disease. Am J Med Genet C Semin Med Genet 2010; 154C: 317-20.

41 Ubeda F, Wilkins JF. Imprinted genes and human disease: an evolutionary perspective. Adv Exp Med Biol 2008; 626: 101-15. 